\title{
Research for Adaptive Control of the Pipes Conveying Fluid
}

\author{
B.Chen,Y.Y.Huang, Z.J.Y in \\ ${ }^{1}$ School of Mechanical \& Engineering \\ University of Science \& Technology Beijing \\ Beijing 100083, P.R. China
}

\begin{abstract}
Pipes with supported ends conveying pulsating fluid may lose the dynamic stability due to parametric instability, therefore the piezoelectric actuators are taken as the control actuators here for controlling vibration of pipeline. First, the controlled mathematical models of pipes conveying fluid were established. Second, model reference adaptive control (MRAC) system and MRAC system based on adapti ve gain of the PD were designed. Third, the effects of controllers were verified and the effects of the control parameters on the controllers were analyzed also. During simulation, the time of vibration displacement decaying to zero, also the time and total energy of control input were taken as evaluation indexes. The results of numerical simulation showed that the pipeline vibration could be controlled well by the controllers which were designed by adaptive control strategy.
\end{abstract}

Keywords-pipes conveying fluid; model reference adaptive control (MRAC); piezoelectric actuator

\section{INTRODUCTION}

Pipes conveying fluid have been widely used in many fields of industry. Considering the fluid is pulsatile, pipeline system may lose the stability by parametric resonances, which the pipeline vibration is continuous and the displacement is very large. Because dynamics of pipes conveying fluid are typical nonlinear dynamic behaviours, how to reduce vibration of pipeline has remained one of intense interest to researchers in this field nowadays. At present, the main control methods are PID control, independent modal space control (IMSC) [1, 2], linear quadratic optimal control (LQOC) [3], adaptive control $[4,5]$, robust control [6] and fu zzy control [7], etc.

\section{MODEL OF CONTROLLED PIPELINE SYSTEM}

The pinned-pinned pipe is taken as an example, the model of controlled system is shown in fig. 1. $x$ and $y$ stand for vertical and horizontal displacement, respectively. $K$ and $G$ are equivalent linear and shear stiffness of the foundation, respectively. $U, R$ and $L$ mean average velocity, outside diameter and length of the pipe, respectively. $\xi_{a}$ and $\xi_{b}$ are the locations of the actuators. $h$ and $\varphi$ are the thickness and half envelop angle of the actuators, respectively.
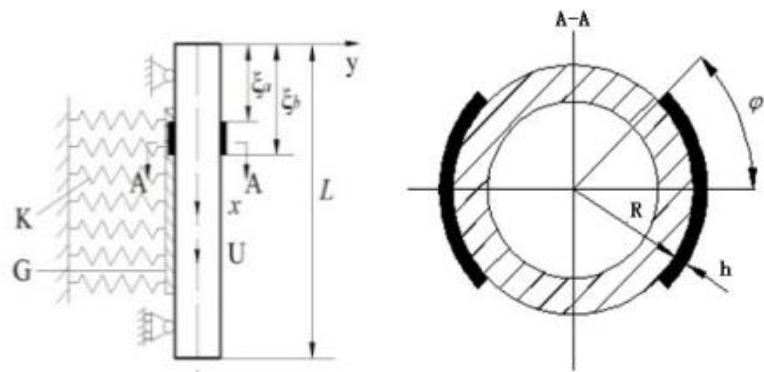

FIGURE I. THE MODEL OF CONT ROLLED SYSTEM

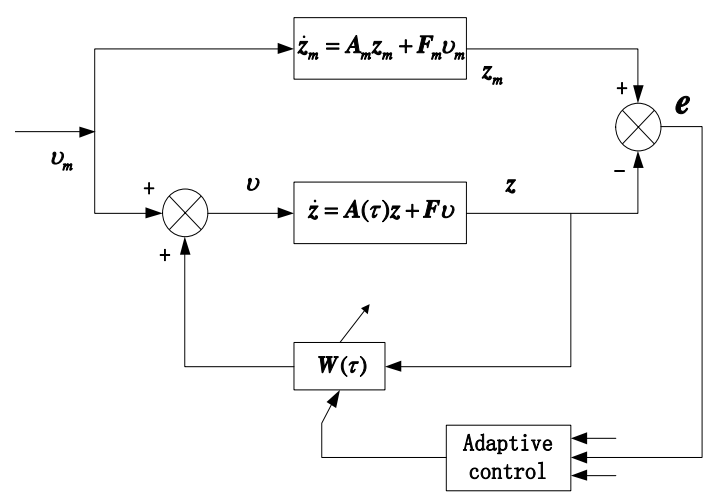

FIGURE II. MRAC SYSTEM

There are two actuators located at opposite side of the pipe and these two actuators are driven $180^{\circ}$ out of phase, the moment could be express as the following expression [8]

$$
M=\frac{4 \psi E_{A} d\left[(R+h)^{3}-R^{3}\right] V \sin \varphi}{3(1+\psi) h}=C_{m} V
$$

where

$$
\psi=E_{C} I_{C} / E_{A} I_{A}
$$

in which $E_{C}$ and $E_{A}$ are the Elastic Modulus of the pipe and the actuator pair, respectively. $I_{C}$ and $I_{A}$ are the cross-section moment of inertia of the pipe and the actuator pair, respectively. $d$ means the electric strain constant.

Suppose the fluid is non-viscosity and incompressible, the effects of supporting motion and high-order variables could be ignored also, the differential equation of motion for controlled pipeline system could be written as 


$$
\begin{aligned}
& {\left[M U^{2}-\bar{T}+\bar{P} A(1-2 v)+(M \dot{U}-(M+m) g)(L-x)-G\right] \frac{\partial^{2} y}{\partial x^{2}}} \\
& +\left(1+a \frac{\partial}{\partial t}\right) E I \frac{\partial^{4} y}{\partial x^{4}}-\left[\left(1+a \frac{\partial}{\partial t}\right) \frac{E \tilde{A}}{2 L} \int_{0}^{L}\left(y^{\prime}\right)^{2} d x\right] \frac{\partial^{2} y}{\partial x^{2}}+(M+m) \frac{\partial^{2} y}{\partial t^{2}} \\
& +2 M U \frac{\partial^{2} y}{\partial x \partial t}+(M+m) g \frac{\partial y}{\partial x}+K y=\frac{\partial}{\partial x}\left\{C_{m} V\left[\delta\left(x-\xi_{a}\right)-\delta\left(x-\xi_{b}\right)\right]\right\}
\end{aligned}
$$

where $M$ and $m$ stand for mass per unit lengths of the fluid and pipe, respectively. $A$ and $\tilde{A}$ are the cross sectional areas of the fluid and pipe, respectively. $\bar{T}$ is the axial force of the cross section in the bottom of the pipe. $\bar{P}$ is the hydrodynamic force per unit area of cross sectional in the endpoints of the pipe. $v$ is the Poisson's ratio of the pipe. $\delta$ is the function of Dirac delta.

In order to avoid the influence of parameters' units, some parameters are introduced to quantified the equation (3)

$$
\begin{aligned}
& \eta=\frac{y}{L}, \xi=\frac{x}{L}, \tau=\left(\frac{E I}{M+m}\right)^{0.5} \frac{t}{L^{2}}, k=\frac{K L^{4}}{E I}, \beta=\left(\frac{M}{M+m}\right)^{0.5} \\
& \bar{g}=\frac{(M+m) g L^{3}}{E I}, \Gamma=\frac{\bar{T} L^{2}}{E I}, \Pi=\frac{\bar{P} A L^{2}}{E I}, \gamma=\frac{\tilde{A} L^{2}}{2 I}, u=\left(\frac{M}{E I}\right)^{0.5} L U \\
& p=\frac{G L^{2}}{E I}, \alpha=\left(\frac{E I}{M+m}\right)^{0.5} \frac{a}{L^{2}}, x_{a}=\frac{\xi_{a}}{L}, x_{b}=\frac{\xi_{b}}{L}, v=\frac{C_{m} L}{E I} V
\end{aligned}
$$

Meanwhile, the Galerkin method is used for solving the motion equation

$$
\eta(\xi, \tau)=\boldsymbol{\Phi}^{T} \boldsymbol{Q}
$$

in which

$$
\begin{aligned}
& \boldsymbol{\Phi}=\left[\phi_{1}(\xi) \phi_{2}(\xi) \cdots \phi_{i}(\xi) \cdots \phi_{N}(\xi)\right]^{T} \\
& \boldsymbol{Q}=\left[q_{1}(\xi) q_{2}(\xi) \cdots q_{i}(\xi) \cdots q_{N}(\xi)\right]^{T}
\end{aligned}
$$

where $\phi_{i}(\xi)$ are vibration mode functions, $q_{i}(\tau)$ are generalized coordinates.

We could define

$$
\int_{0}^{1} \boldsymbol{\Phi} \boldsymbol{\Phi}^{\prime T} d \xi=\boldsymbol{B}, \int_{0}^{1} \boldsymbol{\Phi} \boldsymbol{\Phi}^{\prime \prime T} d \xi=\boldsymbol{C}, \int_{0}^{1} \xi \boldsymbol{\Phi} \boldsymbol{\Phi}^{\prime \prime T} d \xi=\boldsymbol{D}, \int_{0}^{1} \boldsymbol{\Phi} \boldsymbol{\Phi}^{(4) T} d \xi=\boldsymbol{E}
$$

Assume that the velocity of fluid has the following nodimensional form

$$
u=u_{0}(1+\mu \sin (w \tau))
$$

where $u_{0}$ and $w$ are the average velocity and circle frequency of pulsating fluid, respectively. $u$ is the pulse amp litude of the fluid.

Define the preload of the pipeline

$$
T_{\mathrm{o}}=\Gamma-\Pi(1-2 v)
$$

The first order differential equations of pipeline could be expressed as

in which

$$
\dot{z}=\boldsymbol{A} z+\boldsymbol{N} z+M(z)+\boldsymbol{F} v=A(\tau)+\boldsymbol{F} v
$$

$$
\begin{gathered}
\boldsymbol{A}=\left[\begin{array}{cc}
0 & \boldsymbol{I} \\
-\left[u_{0}{ }^{2}-T_{0}-p-\bar{g}\right] \boldsymbol{C}-k \boldsymbol{I}-\boldsymbol{E}-(\boldsymbol{B}+\boldsymbol{D}) \bar{g} & -\left(\alpha \boldsymbol{E}+2 u_{0} \beta \boldsymbol{B}\right)
\end{array}\right] \\
\boldsymbol{N}=-\left[\begin{array}{cc}
0 & 0 \\
\boldsymbol{C}-\boldsymbol{D} & 0
\end{array}\right] \beta u_{0} \mu w \cos (w \tau)-2 u_{0} \mu\left[\begin{array}{cc}
0 & 0 \\
u_{0} \boldsymbol{C} & \beta \boldsymbol{B}
\end{array}\right] \sin (w \tau)
\end{gathered}
$$

$$
\begin{gathered}
\boldsymbol{M}(\boldsymbol{z})=-\gamma \boldsymbol{z}^{T}\left[\begin{array}{cc}
\boldsymbol{C} & 0 \\
0 & 0
\end{array}\right] z\left[\begin{array}{ll}
0 & 0 \\
\boldsymbol{C} & 0
\end{array}\right] z-2 \alpha \gamma z^{T}\left[\begin{array}{ll}
0 & \boldsymbol{C} \\
0 & 0
\end{array}\right] z\left[\begin{array}{ll}
0 & 0 \\
\boldsymbol{C} & 0
\end{array}\right] z \\
\boldsymbol{z}=\left[\begin{array}{ll}
\boldsymbol{Q} & \dot{\boldsymbol{Q}}
\end{array}\right]^{T} ; \boldsymbol{F}=\left[\begin{array}{lll}
0 \cdots 0 & \cdots & \cdots
\end{array} F_{N}\right]^{T}, F_{i}=\phi_{i}^{\prime}\left(x_{b}\right)-\phi_{i}^{\prime}\left(x_{a}\right)
\end{gathered}
$$

III. RESEARCH FOR THE ADAPTIVE CONTROL ALGORIT HMS

\section{A. Model of Reference Adaptive Control}

MRAC system only needs to establish suitable reference model, the model of MRAC system is shown in fig. 2. The MRAC system is mainly composed of reference model, controllable system and adaptive control controller.

The error vector could be expressed as

$$
\boldsymbol{e}=\boldsymbol{z}_{m}-\boldsymbol{z}
$$

where $\boldsymbol{z}_{m}$ is state vector of reference model.

When the control system gets into a good groove, $\boldsymbol{W}=\boldsymbol{W}(\tau)$.

Define

$$
\boldsymbol{\Psi}=\boldsymbol{W}-\boldsymbol{W}(\tau)
$$

And we could define the Lyapunov function as the following

$$
S=\frac{1}{2}\left[\boldsymbol{e}^{T} \boldsymbol{J} \boldsymbol{e}+\operatorname{tr}\left(\boldsymbol{\Psi} \boldsymbol{\Gamma}^{-1} \boldsymbol{\Psi}^{T}\right)\right]
$$

in which $\boldsymbol{J}$ and $\boldsymbol{\Gamma}$ are positive defin ite symmetric matrixes.

When $\dot{S} \leq 0$, the system is stability. The control law could be expressed as

$$
\dot{\boldsymbol{W}}(\tau)=\boldsymbol{F}^{T} \boldsymbol{J e z}^{T} \boldsymbol{\Gamma}
$$

\section{B. Model of Reference Adaptive Control Based on Adaptive Gain of the PD}

Traditional PID controller has many advantages, e.g. the construction of the controller is simple, and controlling parameters are easy for regulating, but it is difficult for PID controller to satisfy the requirement of real-time control for pipeline system. Also, the MRAC system is only applied to the situation that variables of the system are measurable, which is not reality for industrial applications. Thus, in order to solve the problem, several linear parts of the equation need be decoupled, and then according to the parametric resonances of the system, the MRAC system based on adaptive gain of the PD was designed. The model of the system is similar to the MRAC system.

After decoupling, the equations(9) could be expressed as

$$
\dot{\boldsymbol{\theta}}_{\boldsymbol{i}}=\boldsymbol{\Gamma}_{\boldsymbol{i}} \boldsymbol{\theta}_{\boldsymbol{i}}+\boldsymbol{L}_{i} v_{i} \quad i=1,2
$$

in which $\boldsymbol{\theta}_{\boldsymbol{i}}=\left[\begin{array}{ll}\theta_{2 i-1} & \theta_{2 i}\end{array}\right]^{T}, \boldsymbol{\Gamma}_{i}=\left[\begin{array}{cc}\sigma_{i} & w_{i} \\ -w_{i} & \sigma_{i}\end{array}\right], \boldsymbol{L}_{i}=\left[\begin{array}{ll}L_{2 i-1} & L_{2 i}\end{array}\right]^{T}$,

$i=1,2$

where $\theta_{2 i-1}$ are the generalized coordinates of the pipeline system after decoupling. $w_{i}$ are inherent frequencies of the pipeline system. 
The eigenvalues of the matrix $\boldsymbol{\Gamma}_{i}$ have negative real part, thus there are positive definite matrix $\boldsymbol{P}_{i}$ and $\boldsymbol{Q}_{i}$, which should satisfy the following equation

$$
\boldsymbol{\Gamma}_{i}^{T} \boldsymbol{P}_{i}+\boldsymbol{P}_{i} \boldsymbol{\Gamma}_{i}=-\boldsymbol{Q}_{i} \quad i=1,2
$$

We could define the control law of adaptive controller

$$
v_{i}=k_{1} \theta_{2 i-1}+k_{2} \dot{\theta}_{2 i-1} \quad i=1,2
$$

where $k_{i}$ are parameters of the adaptive controller.

Define the Lyapunov function as the following

$$
V=\frac{1}{2} \varepsilon_{i}^{T} P_{i} \varepsilon_{i}+\frac{1}{2 \lambda_{1}} k_{1}^{2}+\frac{1}{2 \lambda_{2}} k_{2}^{2} \quad i=1,2
$$

where $\varepsilon_{i}=\left[\begin{array}{ll}e_{i} & \dot{e}_{i}\end{array}\right]^{T}, \lambda_{i}$ are arith metic numbers.

When $k_{i}$ are expressed in the following equations, $\dot{V} \leq 0$. The system is stable.

$$
\dot{k}_{1}=\lambda_{1} \theta_{2 i-1}\left(p_{1 i} e_{i}+p_{2 i} \dot{e}_{i}\right), \dot{k}_{2}=\lambda_{2} \dot{\theta}_{2 i-1}\left(p_{1 i} e_{i}+p_{2 i} \dot{e}_{i}\right), i=1,2
$$

where $\left[\begin{array}{ll}p_{1 i} & p_{2 i}\end{array}\right]=\left[\begin{array}{ll}L_{2 i-1} & L_{2 i}\end{array}\right] \boldsymbol{P}_{i}, \quad i=1,2$.

When the first order and combination parametric resonances happened, the energy of the first order model motion is the highest, $i=1$. When the second-order parametric resonance happened, the energy of the second order model motion is the highest, $i=2$.

\section{SIMULATIONAND RESULT S ANALYSIS}

In simulation, the parameters of the system were shown in table 1 . The MRAC controller parameters are $\boldsymbol{P}=\boldsymbol{\Gamma}=100 \boldsymbol{I}$. The parameters of the MRAC controller based on adaptive gain of the PD are $\lambda_{1}=50, \lambda_{2}=400$ and $\boldsymbol{Q}=10 \boldsymbol{I}$.Initial values of vibration are $q_{i}=-0.001, \dot{q}_{i}=0$.

TABLE I. PARAMETERS OF THE SYSTEMWITH SUPPORTED ENDS.

\begin{tabular}{|c|c|c|c|c|c|c|c|c|}
\hline & $T_{0}$ & $p$ & $k$ & $\bar{g}$ & $\gamma$ & $\alpha$ & $u_{0}$ & $\mu$ \\
\hline $\begin{array}{c}\text { Pinned- } \\
\text { pinned }\end{array}$ & 0 & 0 & 0 & 0 & 5000 & 0.005 & 2 & 0.4 \\
\hline $\begin{array}{c}\text { Clamped- } \\
\text { clamped }\end{array}$ & 0 & 0 & 0 & 0 & 5000 & 0.0013 & 4 & 0.2 \\
\hline
\end{tabular}

Fig. 3 and fig. 4 were simulation results of the adaptive controllers for pipes with supported ends, when the first order parameter resonance of the pipeline system happened. We could draw a rough conclusion from the figures that the vibration displacements could be decayed to zero in few seconds by the controllers.
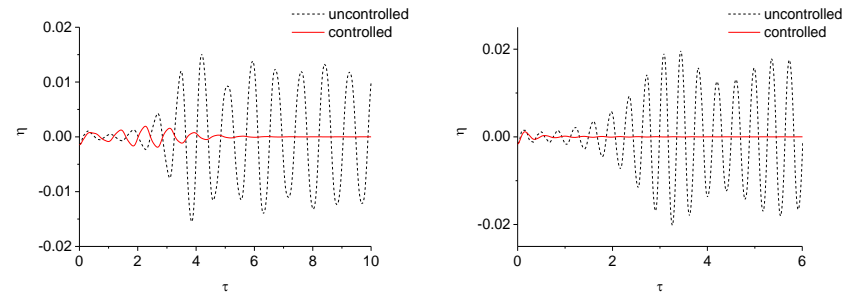

(a) Pinned-pinned pipe. (b) Clamped-clamped pipe

FIGURE III. THE CONT ROL EFFECT S OF MRAC CONT ROLLERS.
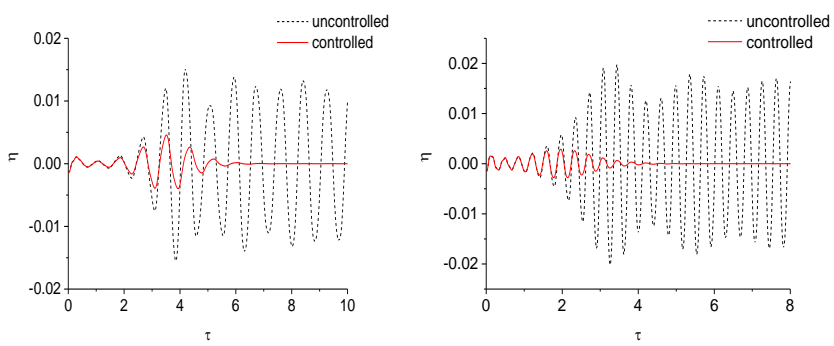

(a) Pinned-pinned pipe. (b) Clamped-clamped pipe

FIGURE IV.THE CONT ROL EFFECT S OF MRAC CONT ROLLERS BASED ON THE ADAPTIVE GAIN OF THEPD.

Because the lengths and locations of the actuators have great influence on the controller, in order to get better control results, the effects of controller parameters on the controllers must be analysed.

The pinned-pinned pipe was taken as the example, the influences of the lengths and locations of the MRAC controllers for control results were shown in fig. 5. We could observe from the figures as below, when the lengths of the actuators were 0.3 and the actuators were located at the midpoint of the pipeline, the displacements of the system were the smallest. Simulation results show that the control effects of the controller were the best.
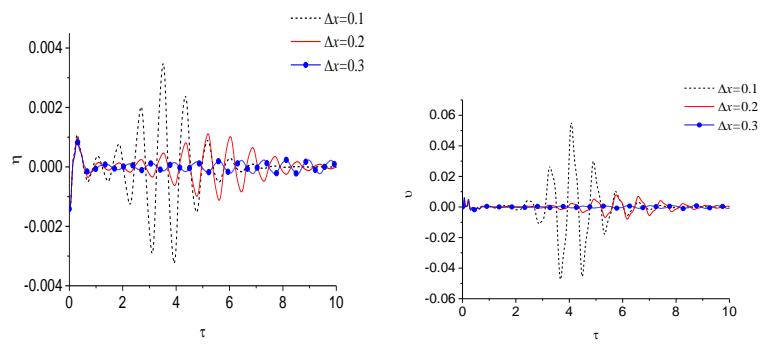

(a) Influences of the lengths of the actuators
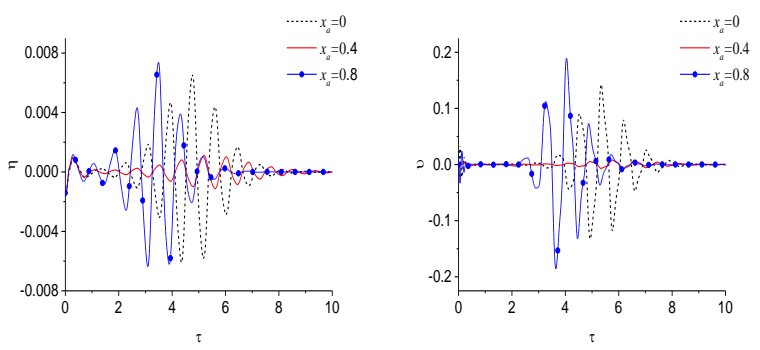

(b) Influences of the locations of the actuat ors

FIGURE V. INFLUENCES OF THE LENGT HS AND LOCAT IONS OF THE MRAC CONTROLLERS.

The influences of the lengths and locations of the MRAC controllers based on the adaptive gain of the PD on the control effects were shown in fig. 6. We could draw similar conclusions from the figures as below. 

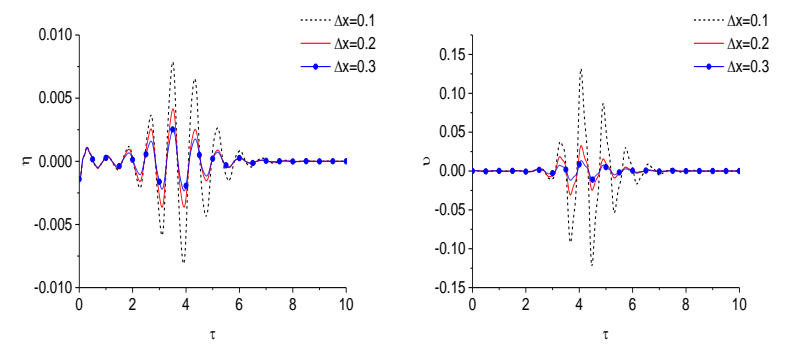

(a) Influences of the lengths of the act uators
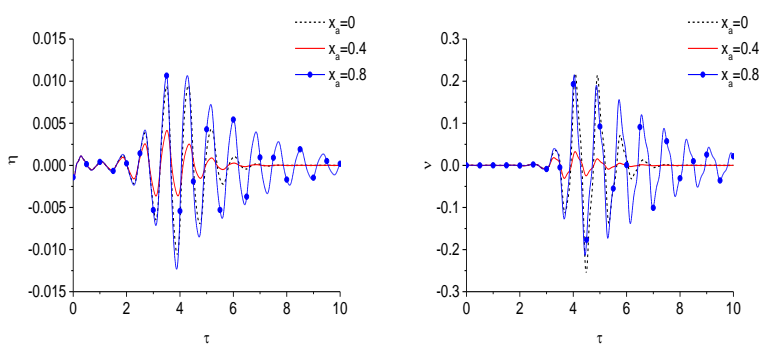

(b) Influences of the locations of the actuat ors

FIGURE VI. PARAMETERS INFLUENCES OF THE MRAC CONT ROLLERS BASED ON THE ADAPTIVE GAIN OFTHEPD.

\section{CONCLUSIONS}

The differential equation of motion for pipes with supported ends has been established. In response to the phenomenon that pipeline system may lose stability due to parametric resonances, the MRAC system and the MRAC system based on the adaptive gain of the PD were designed. The following conclusions could be drawn as following:

First, the vibration displacements of pipes with supported ends can be decayed in short time by the adaptive controllers.

Second, in order to get the best control effects, the lengths and locations of the actuators must be optimized.

Third, compared with the MRAC system and the MRAC system based on the adaptive gain of the PD, the latter can be realized easier than the former in practical applications.

\section{ACKNOWLEDGMENTS}

The authors would like to acknowledge the support of the Fundamental Research Funds for the Central Universities (FRF-SD-12014A).

\section{REFERENCES}

[1] Y.H. Lin \&C.L. Chu, Anew design for independent modal space control of general dynamic system. Journal of Sound and Vibration,180(2), pp.351-361, 1995.

[2] Y.H. Lin\&C.L. Chu, Active model control of Timoshenko pipes conveying fluid. Journal of the Chinese Institute of Engineers, 24(1), pp.65-74, 2001.

[3] Y.B. Sun \& J.B. Qin, Modelling and simulation of piezoelectric smart cantilever beam based on optimal control system. Mechanical Engineering\& Automation, 147(2), pp.59-61, 2008. (In Chinese)

[4] Costa M.H.\&Bermudez C.M., Stochastic Analysis of the Filtered-X LMS Algorithm in Systems with Nonlinear Secondary Paths. IEEE Signal Processing Letters, 11(2), pp.285-288, 2004.

[5] K.F. Zhang, Adaptive control of vibration of a Clamped-Clamped pipe conveying pulsating fluid. Machinery Design \& Manufacture, (12), pp. 149-151, 2008. (In Chinese)

[6] X.B. Liu, H.Y. Su, B.Yao\& J. Chu, Adaptive robust control of nonlinear systems with dynamic uncertainties. International Journal of Adaptive Control and Signal Processing, 23(4), pp. 353-377, 2009.

[7] Y.M. Li, S.C. Tong \& T.S.Li, Adaptive fuzzy output-feedback cont rol for output constrained nonlinear systems in the presence of input saturation. Fuzzy sets and systems, 248, pp. 138-155, 2014.

[8] Y.H. Lin \&C.L. Chu, Comments on "Active model control of vortexinduced vibrations of a flexible cylinder".Journal of Sound and Vibration,175(1), pp. 135-137, 1994. 